
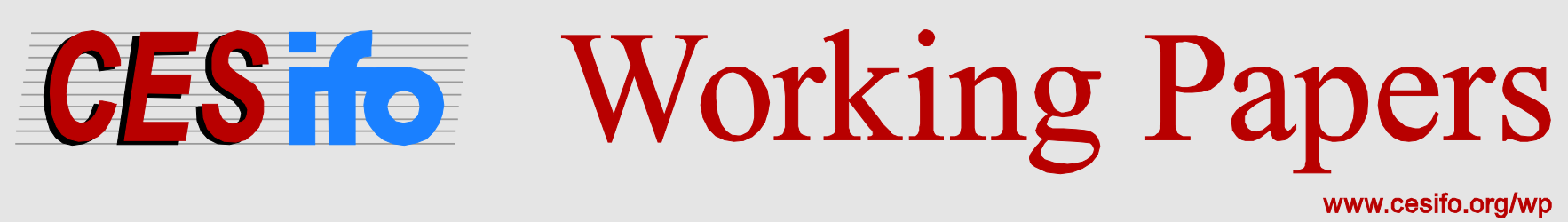

\title{
Psychologically-Based Voting with Uncertainty
}

\author{
Arianna Degan \\ Ming Li
}

CESIFO WORKING PAPER NO. 5014

CATEgory 2: Public CHOICE

OCTOBER 2014

An electronic version of the paper may be downloaded

- from the SSRN website:

- from the RePEc website:

- from the CESifo website:

WWW.SSRN.com

www.RePEc.org

www.CESifo-group.org/wp

\section{CESifo}




\title{
Psychologically-Based Voting with Uncertainty
}

\begin{abstract}
We analyze a psychologically-based model of voter turnout in an election with common value and uncertainty. Our model yields distinctive comparative statics results. First, an increase in the proportion of informed citizens may cause the winning margin for the right candidate to either rise or fall. In addition such increase can have a non-monotonic effect on turnout of uninformed citizens. Second, as the prior becomes more favourable towards the ex ante favoured candidate, turnouts of informed and uninformed voters both change in a nonmonotonic way. Furthermore, total turnout can be positively or negatively correlated with winning margins.
\end{abstract}

JEL-Code: D720.

Keywords: regret, voter turnout, behavioural political economy, psychology and economics, uninformed voters, informed voters.

\author{
Arianna Degan \\ École des Sciences de la Gestion \\ University of Québec at Montréal \\ CP 8888, Succursale Centre-Ville \\ Canada - Montréal, H3C 3P8, Québec \\ degan.arianna@uqam.ca
}

\author{
Ming $\mathrm{Li}$ \\ Concordia University \\ Department of Economics \\ 1455 Boulevard de Maisonneuve Ouest \\ Canada, Montréal, H3G 1M8, Québec \\ ming.li@concordia.ca
}

August, 2014

We thank audiences at Université Laval, Public Choice Society Meetings-Las Vegas, and Workshop in Behavioural Political Economy at CESifo Venice Summer Institute 2014, for helpful comments. We are grateful to Arnaud Dellis, Urs Fischbacher, Frédéric Koessler, and Rebecca Morton for insightful suggestions. We acknowledge the valuable input of Dipjyoti Majumdar in the early stages of the project. All remaining errors are our own. Degan acknowledges, for the early stages of this work, financial support by the Fonds de Recherche sur la Societé et la Culture (FQRSC) programme nouveaux chercheurs. 


\section{Introduction}

Elections are a cornerstone of democracy. How people vote and who go to vote determine how representative the electoral outcome is of preferences of the citizenry. Despite the unanimity in the economics and political science literature about the importance of understanding what motivates participation and voting in elections, this issue remains largely unresolved and is a subject of a huge open debate. In this paper, we build on our previous work (Degan and Merlo [9] and Li and Majumdar [22]) and offer a model of voter turnout based on regret. Our aim is to provide a tractable framework, taking advantage of evidence from psychological studies on decision making and stylized facts about voter turnout. Our model generates distinctive potentially testable implications and can be used in future research to study information provision and acquisition as well as different policy questions.

The standard "rational choice" approach to voter turnout, represented by the pivotalvoter model (see, e.g., Ledyard [20]; Palfrey and Rosenthal [26]; Feddersen and Pesendorfer [10]; Borgers [4]) has apparently been rejected by empirical and experimental evidence in a variety of dimensions (see, e.g., Levine and Palfrey [21]; Coate, Conlin, and Moro [7]; Agranov et al. [1]). As a response, models of turnout have been developed that have deviated from the assumption that individuals are fully rational and take only into consideration the probability of being pivotal when deciding whether and how to cast a vote. All alternative approaches, which take away the assumption that individuals vote solely to directly affect the result of the elections, are inevitably based on some behavioural assumption.

Rule or group utilitarian models of turnout (see, e.g., Harsanyi [16], Feddersen and Sandroni [11, 12], Coate and Conlin [6]) assume that individuals are ethical in the sense that they follow the rule that if followed by everybody else in the population (or their group) maximizes the welfare of the population (or the group to which they belong). Expressive voting models (see, e.g., Fiorina [14]; Aldrich [2]; Schuessler [30]; Hillman [17, 18]; Degan [8]; Ortoleva and Snowberg [25]) assume that individuals benefit from voting due to the utility they derive from self-expression. ${ }^{1}$ Biased-beliefs models (see, e.g., Ortoleva and Snowberg [25]; Chaves and Peralta [5]) assume that citizens have one of possible cognitive biases. Ortoleva and Snowberg [25] assume that citizens are overconfident due to correlation neglect. Chaves and Peralta [5] assume that citizens are self-delusive, in the sense that they believe that an exogenous proportion of like-minded citizen behave like them. Regret-based theories of voting (see, e.g., Ferejohn and Fiorina [13]; Degan and

\footnotetext{
${ }^{1}$ Hamlin and Jennings [15] provide a comprehensive survey of the literature on expressive political behaviour.
} 
Merlo [9]; Li and Majumdar [22]) are based on the behavioural assumption that individuals are concerned about making the wrong decision and experience regret if they fail to vote or vote for the wrong option. Ferejohn and Fiorina [13] postulate that citizens may vote in order to avoid the regret they would experience if they were to abstain in a situation where their vote would have been decisive. So, although their citizens are not able to calculate the probability of being pivotal, they think that their action could affect the result of the election. Degan and Merlo [9] consider a context where citizens are uncertain about who the "right" candidate is and incur a psychological cost upon the act of voting associated with the possibility of voting for the wrong candidate. Li and Majumdar [22] consider a context with heterogeneous preferences and certainty where voters experience regret if they fail to vote or vote for the wrong candidate and such regret depends on the margins of victory. Similar to Degan and Merlo [9], any instrumental motivation is completely absent. Different from Degan and Merlo [9], who assume that the disutility for possibly making the wrong choice is experienced upon voting, both Ferejohn and Fiorina [13] and $\mathrm{Li}$ and Majumdar [22] assume that voters make voting decisions based on anticipated ex-post regret. ${ }^{2,3}$

There appears to be empirical support for the common underlying assumptions of regret-based models of voting. For example, CBS News and the New York Times conducted a poll of 1720 Americans during November 10-12, 2000 and found that 55\% of those who did not vote regretted not voting in the 2000 U.S. presidential elections. In France, following the surprising elimination of the socialist candidate Lionel Jospin at the hands of the far-right anti-immigration candidate Jean-Marie Le Pen in the first round of the 2002 French presidential election, many French voters regretted their decision not to vote. ${ }^{4}$ In these (large) elections, each individual voter's decision would not have affected the outcome of the election, yet voters who did not vote experienced regret afterwards. Similar results are recurrent in a variety of electoral surveys. Degan and Merlo [9] find that their regret-based model of election is able to explain the observed turnout and voting patterns in US presidential and House elections.

Besides the above-cited evidence specific to behaviour in elections, regret is a widely observed psychological phenomenon (see Landman [19]). In psychological research, regret has increasingly become recognized as an important factor in decision making. ${ }^{5}$ Some

\footnotetext{
${ }^{2}$ For a discussion and justifications of regret-based voting theories see, e.g. Li and Majumdar [22] and Degan and Merlo [9].

${ }^{3}$ Merlo [24] provides a concise survey of the different models of voter turnout, while Schnellenbach and Schubert [29] discuss more of the models with psychological origins.

${ }^{4}$ Financial Times, London, May 4, 2002, p. 09. These two examples are borrowed from Li and Majumdar [22].

${ }^{5}$ Roese and Olson [28] provide an anthology of research in counterfactual thinking, while Roese [27]
} 
economic and decision theorists have emphasized the role of anticipated regret in decision making (Loomes and Sugden [23]; Bell [3]). However, the concept of regret in these models is very narrowly defined and cannot easily be applied across different situations.

In this paper, we present a model of voter participation and voting in large elections based on anticipated ex-post regret. As anticipated, our model builds on those of Degan and Merlo [9] and Li and Majumdar [22]. ${ }^{6}$ A key assumption is that potential voters experience regret if they fail to vote or vote for the wrong candidate. Such regret is dependent on who wins the election and what the margin of victory is. In particular, a voter experiences more regret if the "wrong" candidate wins, and experiences more regret if the election outcome is close. That people experience regret when they abstain is consistent with the long standing idea that failure to vote is viewed negatively in a democratic society. It is conceivable that such negative (self-)perception is especially strong when the election is close or when the "right" candidate loses the election. One possible interpretation is that citizens are ethical. They believe that by failing to vote or voting for the wrong candidate, they would disappoint people with similar political preferences to themselves and be subject to blame, hence they will experience regret from their decision. The psychological effect of the letdown will be stronger, and therefore regret higher, when the election result is close and when the election outcome is against the citizen's preferences.

In our model, there are two candidates, $\mathrm{A}$ and $\mathrm{B}$, and two states of the world, $s_{A}$ and $s_{B}$. There are a continuum of voters with common preferences. They prefer candidate $A$ in state $s_{A}$, which occurs with probability $p$ and candidate $B$ in state $s_{B}$, which occurs with probability $1-p$. A proportion of citizens are informed about the state, while the remaining are not. Each citizen's utility is determined by his anticipated regret from (not) voting or voting for the wrong candidate, and a cost of voting, which is drawn from a random distribution. He votes for a candidate if doing so gives him the highest expected utility among the three options available (voting for $A$, for $B$, or abstaining).

We find that in any equilibrium uninformed voters must vote for the ex-ante preferred candidate at least with probability $1 / 2$. It follows that the right candidate is always elected in the more likely state. We characterize the equilibrium and find conditions for a desirable equilibrium, where the right candidate is also elected in the less likely state, and for an undesirable equilibrium, where the right candidate is not elected in the less likely state. Undesirable equilibria exist for relatively low proportion of informed citizens. Mixing equilibria exist when the probabilities of the two states are close.

writes about the relation between regret and decision making to the general readership.

${ }^{6}$ As do Degan and Merlo [9], but not Li and Majumdar [22], we assume that a proportion of citizens are uncertain about who the "righ" candidate is. 
Our model generates both predictions in line with previous models of turnout and others that are novel or depart from them.

First, in all equilibria informed citizens have a higher turnout than uninformed ones. Furthermore, regret in the less likely state is higher than in the more likely one, which in turns implies that informed voters turn out more in the less likely state - a version of the "underdog effect."

Second, as it becomes increasingly clear who the best candidate is, the margin of victory for the best candidate always increases, which is intuitive. Furthermore, and interestingly, as information becomes more clear, the change in turnouts of both uninformed and informed citizens is non-monotonic. In particular, turnout of uninformed citizens initially decreases but then increases. The turnout of informed citizens changes in a more complex way. It always decreases in the ex-ante more likely state, and it increases in the other state if there are enough informed citizens so that the right candidate also wins in the other state. Otherwise, it changes non-monotonically: it first increases and then decreases.

Third, when the proportion of informed citizens increases, the margin of victory for the more likely candidate increases in all types of equilibria except the undesirable mixing equilibrium, while that for the less likely candidate always increases. In the meantime, the turnout of informed citizens decreases in all cases but in the less likely state in the undesirable pure equilibrium, while that of uninformed voters decreases except in the undesirable mixing equilibrium.

We also use the above comparative statics to assess the relationship between the margin of victory and total turnout. When we vary the degree of clarity about the information on who the right candidate is, holding everything else fixed, we find that higher (absolute) margin can correspond to higher or lower turnout, depending on the type of equilibrium, the realization of the state, and the proportion of informed citizens. Similar results about correlation between margin of victory and turnout can be obtained if we vary the proportion of informed citizens, holding everything else fixed.

A key modelling assumption we adopt is that margins of victory affect citizens' utility in a non-instrumental way. The way they affect turnout resembles the way winning margins indirectly affect turnout in pivotal-voter models, including that of Feddersen and Pesendorfer [10]. ${ }^{7}$ We show however that our model, where citizens do not take

\footnotetext{
${ }^{7}$ In pivotal-voter models, there is a negative relationship between expected margins of victory and pivotal probabilities. Among existing pivotal-voter models we take as a benchmark for comparison the one of Feddersen and Pesendorfer [10] because, despite the absence of costly voting, it is the one that share our modelling assumptions the most. Namely, it is a common value setting with informed and uninformed citizens.
} 
the probability of being pivotal into account and where positive and negative margins of victory affects utility differently, delivers important different predictions from Feddersen and Pesendorfer [10]. First, in our model, even in large elections there is not necessarily information aggregation, as we find undesirable equilibria where only the ex-ante favoured candidate always wins in the right state, but the non-favoured candidate always loses. This result is not that surprising because our voters do not condition on the probability of being pivotal when they make a voting decision but they are only concerned about the psychological utility related to the margins of victory. Second, in Feddersen and Pesendorfer's [10] model, in equilibrium uninformed citizens either vote for one candidate or abstain, but they never mix between the two candidates, as they do in our model. Third, their model predicts that turnout of uninformed citizens always increases as the proportion of informed voters increases. In our model, this happens only in undesirable mixed-strategy equilibria. Fourth, in Feddersen and Pesendorfer's [10] model, the margin of victory increases with the proportion of people that are informed, while in our model this is not the case in various circumstances. Finally, in our model the ex-ante probability of the right state has an important impact on both turnout and voting decisions. ${ }^{8}$

To summarize, we make the following contributions in this paper: we provide a tractable framework that is on solid footing in terms of consistency with psychological studies on decision making and stylized facts about voter turnout. We deliver in this model many interesting distinctive comparative statics results, which provide an opportunity to test the validity of the model using experimental or empirical studies. In addition, given its inclusion of uncertainty, the model can be used, in future research, to study information provision and acquisition as well as other policy questions.

The paper is organized as follows. In section 2 we present the model. In section 3 we analyze the equilibrium. In section 4 we conduct comparative statics analysis and in section 5 we conclude.

\section{$2 \quad$ Model}

In our model, there are two candidates, $A$ and $B$. There are a continuum of citizens with common preferences. They prefer the candidate suitable to the state of the world: candidate $A$ in state $s_{A}$ and candidate $B$ in state $s_{B}$. State $s_{A}$ occurs with probability $p$ and state $s_{B}$ with probability $1-p$. Without loss of generality, we focus on the case $p \geq 1 / 2$.

Since there are a continuum of citizens, the probability of any voter being pivotal is

\footnotetext{
${ }^{8}$ Feddersen and Pesendorfer [10] write that, when the population is large, "One parameter that does not play a critical role in either the decision to participate or voting choice is the common knowledge prior belief $(\alpha)$ concerning the state of the world ..."
} 
zero. On the other hand, a citizen incurs regret if he abstains or votes for the "wrong" candidate, that is, the candidate who is not suitable to the state of the world.

Following Li and Majumdar [22], we assume a voter's regret is $r(m)$, when the margin of victory is $m$ (or, margin of loss if $m<0$ ) for the "right" candidate. For notational convenience, we divide the regret function into two parts: $r_{-}: \mathbb{R}_{-} \rightarrow \mathbb{R}_{+}$and $r_{+}: \mathbb{R}_{+} \rightarrow$ $\mathbb{R}_{+}$, defined as follows

$$
\begin{aligned}
& r_{-}(m)=r(m) \text { for } m<0 ; \\
& r_{+}(m)=r(m) \text { for } m>0 ;
\end{aligned}
$$

We assume that $r$ has the following property.

Assumption 1. The regret function $r$ is nonnegative, differentiable, and satisfies the following assumptions:

(i) $r$ is strictly increasing for $m<0$ and strictly decreasing for $m>0$ up to $\bar{m}$ as defined below;

(ii) $r(-1)=r(1)=0$;

(iii) Winner Regrets Less:

$$
r_{+}(m)<r_{-}(-m) \text { for all } m>0
$$

(iv) $r_{+}(\bar{m})=0$ for $m \geq \bar{m}$, for some $\bar{m} \leq 1$.

Part (i) means that regret is stronger when the outcome of the election is close. Part (ii) says that regret reaches zero when the margin of victory of a candidate becomes large. Part (iii), the winner-regret-less assumption, means that, for a given margin of victory, an abstaining voter's regret is higher when his favoured candidate loses, than that when his favoured candidate wins by the same margin. This is also true for very arbitrarily small margins. Part (iv) is made for simplicity. ${ }^{9}$

The state is not observed by all citizens. A fraction $q \in(0,1)$ of the population is informed and observes the state, while the remaining fraction $1-q$ is uninformed and only knows its probability distribution. In addition, a citizen incurs a cost $c$ when he participates in an election. Each citizen's cost of participation is indepedently drawn from a random distribution with a continuous distribution function $F$ and density function $f$.

Table 1 summarizes citizens' preferences conditional on the state of the world, $s_{I}$, $I=\{A, B\}$.

\footnotetext{
${ }^{9}$ Our Winner Regrets Less assumption is a weaker version of the one made by Li and Majumdar [22], who assume that $\frac{r_{-}^{\prime}(-m)}{-r^{\prime}(m)}<1$ for $m>0$. Part (iv) of our Assumption 1 would hold necessarily if, in addition to assuming continuity of the regret function, we were making their stronger assumption.
} 


\begin{tabular}{lll} 
& State $s_{A}$ & State $s_{B}$ \\
\hline Abstain & $-r\left(m_{A}\right)$ & $-r\left(m_{B}\right)$ \\
Vote for $A$ & $-c$ & $-c-r\left(m_{B}\right)$ \\
Vote for $B$ & $-c-r\left(m_{A}\right)$ & $-c$ \\
\hline
\end{tabular}

Table 1: Citizens' preferences

When deciding whether to participate and, eventually how to vote, a citizen has to compare the expected utility of the three options: abstain, voting for $A$, and voting for $B$. Table 2 shows the uninformed citizen's expected utility for each option: where $1\left\{s_{I}\right\}$ is an

\begin{tabular}{cc} 
& Expected Utility \\
\hline Abstain & $-E\left[1\left\{s_{A}\right\} r\left(m_{A}\right)+1\left\{s_{B}\right\} r\left(m_{B}\right)\right]$ \\
Vote for $A$ & $-c-E\left[1\left\{s_{B}\right\} r\left(m_{B}\right)\right]$ \\
Vote for $B$ & $-c-E\left[1\left\{s_{A}\right\} r\left(m_{A}\right)\right]$
\end{tabular}

Table 2: Uninformed citizens' choices

indicator function that takes value 1 when the state of the world is $s_{I}$ and zero otherwise. To facilitate analysis, we normalize a citizen's payoff by adding $c+E\left[1\left\{s_{A}\right\} r\left(m_{A}\right)+\right.$ $\left.1\left\{s_{A}\right\} r\left(m_{B}\right)\right]$ to each expected utility value in Table 2. Consequently, an uninformed citizen's problem can be represented as

$$
\max \left\{c, E\left[1\left\{s_{A}\right\} r\left(m_{A}\right)\right], E\left[1\left\{s_{B}\right\} r\left(m_{B}\right)\right]\right\},
$$

with utility values representing abstaining, voting for $A$, and voting for $B$ respectively.

Given anticipated margins of victory in the two states, $m_{A}$ and $m_{B}$, an uniformed citizen with cost of voting $c$ votes (for either $A$ or $B$ ) if and only if

$$
c \leq \min \left\{\operatorname{pr}\left(m_{A}\right),(1-p) r\left(m_{B}\right)\right\} .
$$

An informed citizen, in contrast, will never choose to vote for the wrong candidate. He votes for the right candidate as long as his voting cost is relatively low, otherwise he abstains. An informed citizen with cost $c$ votes in state $s_{I}$ if and only if

$$
c \leq r\left(m_{I}\right),
$$

where $m_{I}$ is the winning margin of candidate $I$ in state $s_{I}$, where $I=A, B$. 


\section{Equilibrium characterization}

In this section, we characterize the equilibrium of our model. Let $\tau_{u}$ denote turnout of uninformed citizens and $\alpha \in[0,1]$ the probability that an uninformed citizen votes for candidate $A$, conditioning on voting. Similarly, let $\tau_{I}$ denote turnout of informed citizens in state $I=A, B$. As we have argued in the previous section, an informed citizen votes if and only if his cost is lower than the regret of not voting, which implies that $\tau_{I}=F\left(r\left(m_{I}\right)\right)$.

In equilibrium, we must require that the realized margins of victory of the two candidates be consistent with the anticipated margins of victory $m_{A}$ and $m_{B}$ and the behaviour they induce:

$$
\begin{aligned}
& m_{A}=q F\left(r\left(m_{A}\right)\right)+(1-q) \tau_{u}(2 \alpha-1), \\
& m_{B}=q F\left(r\left(m_{B}\right)\right)+(1-q) \tau_{u}(1-2 \alpha) .
\end{aligned}
$$

Summing the two equations, we obtain

$$
m_{A}+m_{B}=q\left[F\left(r\left(m_{A}\right)\right)+F\left(r\left(m_{B}\right)\right)\right]
$$

We may make two simple observations from (3). First, the sum of the winning margins for the right candidate in each state is equal to the sum of the turnouts of the informed citizens. The reason is that uninformed citizens cannot condition their votes on the state, so that if they increase the winning margin of the right candidate in one state, they must decrease it in the other state. Second, the left hand side of (3) is the sum of the anticipated margins, while the right hand side is the sum of realized margins given the anticipated margins. In equilibrium, they must be equal. We may also rewrite it as

$$
\left[m_{A}-F\left(r\left(m_{A}\right)\right)\right]+\left[m_{B}-q F\left(r\left(m_{B}\right)\right)\right]=0 .
$$

The interpretation is that the number of votes from the uninformed voters needed to make up for the shortfall between the anticipated margin of Candidate $A$ and the realized margin due to votes for $A$ cast by the informed voters in state $A$, must be exactly the opposite of the counterpart for Candidate $B$.

From (3), we may obtain some immediate but important implications, summarized in the following Lemma, about the winning margins in the two states.

Lemma 1. In all voting equilibria, $m_{A}>0$ and $m_{A} \geq m_{B}$.

Proof of Lemma 1: First, we show that $m_{A}>0$. According to (3), $m_{A}+m_{B}>0$. Thus, we may rule out the case where $m_{A} \leq 0$ and $m_{B} \leq 0$. Furthermore, $m_{A} \leq 0$ 
implies that there must be uninformed voters who vote for $B$. But, an uninformed voter finds it optimal to vote for $B$ only if $(1-p) r\left(m_{B}\right) \geq \operatorname{pr}\left(m_{A}\right)$. By the assumptions of "winner regrets less" and $p \geq 1 / 2$, we must have $0<m_{B} \leq-m_{A}$, which would imply $m_{A}+m_{B} \leq 0$, a contradiction. Hence, we conclude $m_{A}>0$. To show $m_{A} \geq m_{B}$, assume to the contrary, $m_{B}>m_{A}>0$. This implies $\operatorname{pr}\left(m_{A}\right)>(1-p) r\left(m_{B}\right)$, which in turn implies all uninformed voters who participate in the election vote for $A$, i.e. $\alpha=1$. Thus,

$$
m_{A}=q F\left(r\left(m_{A}\right)\right)+(1-q) \tau(2 \alpha-1)>q F\left(r\left(m_{B}\right)\right)+(1-q) \tau(1-2 \alpha)=m_{B}
$$

by (1) and (2), a contradiction.

Lemma 1 states that in the ex-ante more likely state $\left(s_{A}\right)$, the right candidate $(A)$ wins. In the other state $\left(s_{B}\right)$, the right candidate $(B)$ either wins by a smaller margin or loses the election. The informed voters always vote for the right candidate. Thus, in either state, if the right candidate loses the election, it must be because more uninformed voters have voted for the wrong candidate than for the right candidate. Since the strategies of uninformed voters do not depend on the state, it must mean more uninformed voters have voted for the right candidate in the other state, which guarantees that in that state the right candidate wins. However, since state $s_{A}$ is more likely and winners regret less, if $A$ loses the election in state $s_{A}$, in order for uninformed voters to be willing to vote for $B$, it must mean that in state $s_{B}$, candidate $B$ wins by a smaller margin than $A$ 's margin of loss, which implies the sum of the two margins is negative. But this is impossible, as their sum is simply equal to the sum of the turnouts of informed voters in either state. Thus, candidate $A$ must win the election in state $s_{A}$. In addition, if in both states the right candidate wins the election, $A$ 's winning margin has to be higher, because if $B$ had a higher winning margin then an uninformed voter would strictly prefer to vote for $A$ and informed voters would vote for $A$ more than they vote for $B$, which results in a contradiction.

Lemma 1, (1), and (2) also have implications on the mixing probabilities of uninformed voters, as the following lemma demonstrates.

Lemma 2. In all voting equilibria, $\alpha \geq 1 / 2$.

Proof of Lemma 2: Assume, to the contrary, $\alpha<1 / 2$. This implies that the uninformed voters vote for $B$ with a positive probability. Hence,

$$
\operatorname{pr}\left(m_{A}\right) \leq(1-p) r\left(m_{B}\right) .
$$

Since $p \geq 1 / 2, r\left(m_{A}\right) \leq r\left(m_{B}\right)$. Using (1) and (2), we conclude $m_{B}>m_{A}$, a contradiction to Lemma 1. 
The above lemma implies that there cannot be any equilibrium in which the uninformed voters vote only for $B$. This leaves us with two types of equilibria in terms of the uniformed voters' behaviour, namely, pure, in which they always vote for $A$ and, mixing, in which they are split between $A$ and $B$. In addition, this implies that the turnout for uninformed citizens can be written as

$$
\tau_{u}=F\left(\operatorname{pr}\left(m_{A}\right)\right)
$$

While in state $s_{A}$ the right candidate always wins (see Lemma 1), in state $s_{B}$ the wrong candidate could be elected. Without loss of generality, with the only purpose of simplifying exposition, we assume that when the margin of victory is zero, candidate $B$ is elected. We distinguish between two types of equilibria: desirable and undesirable.

Definition 1. An equilibrium is called desirable when $m_{A}>0$ and $m_{B} \geq 0$.

Definition 2. An equilibrium is called undesirable if $m_{A}>0$ and $m_{B}<0$.

In terms of the election outcome, desirable equilibria are those where the right candidate is elected in both states, while in undesirable equilibria candidate $B$ is never elected. In addition, we will divide our analysis according to whether the uninformed voters mix between voting for two candidates..$^{10,11}$

Definition 3. An equilibrium is called mixing if uninformed voters mix between voting for $A$ and voting for $B$.

In the following two subsections we analyze in details mixing and desirable purestrategy and undesirable equilibria.

\subsection{Mixing equilibria}

In a mixing equilibrium both candidates receive votes from uninformed voters. We first consider desirable and then undesirable mixing equilibria.

\footnotetext{
${ }^{10}$ Note that we can abstract from uninformed voters that are indifferent between abstention and participation, because for any anticipated margins of victory this indifference happens only for a particular realization of the cost, which has zero probability measure.

${ }^{11}$ Since we have a continuum of voters, a "mixin" equilibrium can be equivalently realized through either each uninformed voter playing a mixed strategy or a subset of voters voting for $A$ and the remaining voting for $B$ (i.e., all voters playing pure strategies).
} 


\subsubsection{Desirable mixing equilibrium}

In a desirable mixing equilibrium the right candidate is elected in both states and both candidates receive votes from uninformed voters. In order for this to occur, we need uninformed voters to be indifferent between voting for $A$ and for $B$.

$$
\operatorname{pr}\left(m_{A}\right)=(1-p) r\left(m_{B}\right) .
$$

Let $m_{0} \in[0,1]$ be defined by

$$
\operatorname{pr}\left(m_{0}\right)=(1-p) r(0) .
$$

In words, $m_{0}$ is the margin of victory for candidate $A$ in state $s_{A}$ that would make the uninformed voter indifferent between $A$ and $B$, when in state $s_{B}$, the margin of victory for candidate $B$ is 0 , which is the lowest margin she can get in a desirable equilibrium. Note that $m_{0}$ is increasing in $p$, equal to 0 when $p=1 / 2$, and equal to $\bar{m}$ when $p=1 .^{12}$

For each $m_{A} \in\left[m_{0}, 1\right]$, there exists a unique $m_{B} \geq 0$ that satisfies (5). With a slight abuse of notation, we use $m_{B}\left(m_{A}\right)$ to denote this value (we have suppressed its dependence on $p$ ).

Lemma 3. The function $m_{B}(\cdot)$ satisfies the following properties:

1. $m_{B}(\cdot)$ is increasing in $m_{A}$ (weakly when $\left.m_{A} \geq \bar{m}\right)$;

2. $m_{B}\left(m_{0}\right)=0$ and $m_{B}\left(m_{A}\right)=\bar{m}$, for all $m_{A} \geq \bar{m}$;

3. $m_{B}(\cdot)$ is decreasing in $p$.

Proof of Lemma 3: Part 2 is directly implied by our assumptions on $r$ and the definition of $m_{0}$. By (5),

$$
\begin{aligned}
\frac{\partial m_{B}}{\partial p} & =\frac{r\left(m_{B}\right)+r\left(m_{A}\right)}{(1-p) r^{\prime}\left(m_{B}\right)}<0, \\
\frac{\partial m_{B}}{\partial m_{A}} & =-\frac{p r^{\prime}\left(m_{A}\right)}{-(1-p) r^{\prime}\left(m_{B}\right)}>0,
\end{aligned}
$$

where the signs come from our assumption that $r^{\prime}(m)<0$ for $m>0$.

Thus, (3) becomes

$$
m_{A}+m_{B}\left(m_{A}\right)=q\left[F\left(r\left(m_{A}\right)\right)+F\left(r\left(m_{B}\left(m_{A}\right)\right)\right)\right] .
$$

\footnotetext{
${ }^{12}$ To be more precise, when $p=1$, any $m_{0} \geq \bar{m}$ satisfies (6) but only $\bar{m}$ will be relevant.
} 
Observe that the left hand side of the above equation is continuous and increasing in $m_{A}$ and the right hand side is continuous and decreasing. In addition, as $m_{A}$ increases to $\bar{m}$, the left hand side increases to $2 \bar{m}>0$, while the right hand side decreases to 0 . So, in order for a solution $m_{A}^{*} \in\left[m_{0}, \bar{m}\right]$ to the above equation to exist, it is necessary and sufficient that

$$
m_{0}+0 \leq q\left[F\left(r\left(m_{0}\right)\right)+F(r(0))\right]
$$

or

$$
m_{0}-q F\left(r\left(m_{0}\right)\right) \leq q F(r(0)) .
$$

We now analyze under which conditions (8), which assures existence of margins of victory consistent with both the mixing condition (5) and the coherency condition (7) between anticipated and realized margins, holds. When $p=1 / 2$, we have $m_{0}=0$, so the above condition is satisfied for all $q$. When $p$ increases to $1, m_{0}$ increases to $\bar{m}$ so $m_{0}-q F\left(r\left(m_{0}\right)\right)=\bar{m}$. Condition (8) is violated for $q<\bar{m} / F(r(0))$. Observes that the left hand side is increasing in $m_{0}$. By monotonicity and continuity, for any given $q$, there exists a $\bar{p}(q) \in[1 / 2,1]$ such that $(8)$ is satisfied for all $p \leq \bar{p}(q)$. In particular, $\bar{p}(q)=1$ for $q \geq \bar{m} / F(r(0))$ and $\bar{p}(q)$ is increasing in $q$ for $q<\bar{m} / F(r(0))$.

Before continuing the analysis, it is useful to impose additional assumptions on the regret function and the density $f$ of the cost, and then to introduce some comparative statics results, which will prove useful to complete the characterization of the equilibrium.

Assumption 2. The density function of the cost of voting, $f$, is such that $f(x) x$ is increasing in $x$.

Assumption 3. The regret function, $r$, is such that $r^{\prime}(m) / r(m)$ is decreasing in $m$, for $m>0$. In other words, $r(m)$ is log-concave when $m>0$.

For example, Assumption 2 is satisfied by the uniform distribution and Assumption 3 is satisfied by concave regret functions, including affine ones.

Before proceeding with the analysis of the equilibrium, it is useful to introduce some notation that will be used throughout the paper.

$$
\begin{aligned}
X_{A} & =1-q f\left(r\left(m_{A}\right)\right) r^{\prime}\left(m_{A}\right) \\
X_{B} & =1-q f\left(r\left(m_{B}\right)\right) r^{\prime}\left(m_{B}\right) \\
\Gamma & =\left[r\left(m_{A}\right)+r\left(m_{B}\right)\right] X_{B} \\
\Delta & =(1-p) r^{\prime}\left(m_{B}\left(m_{A}\right)\right) X_{A}+p r^{\prime}\left(m_{A}\right) X_{B}
\end{aligned}
$$

In words, $X_{i}, i=\{A, B\}$, expresses how the difference between anticipated margins for candidate $i$ in state $s_{i}$ and the realized margins for such a candidate due to the votes of 
informed voters is directly affected by a change in the anticipated margin $m_{i}$. The term $\Gamma \cdot r^{\prime}\left(m_{B}\right)$ gives the direct effect of $p$, fixing the margin $m_{A}$, on the difference between the sum of anticipated and realized margins when uninformed voters mix, that is the difference between LHS and RHS in (7) when $m_{B}$ has to adjust so as to maintain the indifference condition (5). The term $\Delta$, for fixed $p$ and $q$, is a function of $m_{A}$ only, as $m_{B}$ must respect the mixing condition (5). Therefore, it should be clear that the term $X_{B}$ in the expression for $\Delta$ is evaluated at $m_{B}=m_{B}\left(m_{A}\right)$. To provide an interpretation for $\Delta$, notice that $\Delta \cdot r^{\prime}\left(m_{B}\right)$ measures how the difference in the sum of anticipated and realized margins is affected by a change in the anticipated margin $m_{A}$, when voters mix.

Applying the implicit function theorem to (7) we have:

$$
\begin{aligned}
\frac{d m_{A}^{*}}{d p} & =-\frac{\Gamma^{*}}{\Delta^{*}}>0, \\
\frac{d m_{A}^{*}}{d q} & =\frac{\left[F\left(r\left(m_{A}^{*}\right)\right)+F\left(r\left(m_{B}^{*}\right)\right](1-p) r^{\prime}\left(m_{B}^{*}\right)\right.}{\Delta^{*}}>0,
\end{aligned}
$$

where $m_{B}^{*}=m_{B}\left(m_{A}^{*}\right)$ and $\Gamma^{*}>0$ and $\Delta^{*}<0$ are the terms defined above evaluated at $\left(m_{A}^{*}, m_{B}^{*}\right)$. Notice, in fact, that for any $m_{i}>0, i=A, B, X_{i}$ is greater than 1 and $r^{\prime}\left(m_{i}\right)<0$.

From the above results we can obtain the comparative statics for $m_{B}^{*}$ :

$$
\begin{aligned}
\frac{d m_{B}^{*}}{d p} & =\frac{\partial m_{B}}{\partial m_{A}} \frac{d m_{A}^{*}}{d p}+\left.\frac{\partial m_{B}}{\partial p}\right|_{m_{A}=m_{A}^{*}}= \\
-\frac{r\left(m_{A}^{*}\right)+r\left(m_{B}^{*}\right)}{(1-p) r^{\prime}\left(m_{B}^{*}\right)}\left[\frac{p r^{\prime}\left(m_{A}^{*}\right) X_{B}}{\Delta^{*}}-1\right] & <0 \\
\frac{d m_{B}^{*}}{d q} & =\frac{\partial m_{B}}{\partial m_{A}} \frac{d m_{A}^{*}}{d q}>0
\end{aligned}
$$

and finally for the turnout of uninformed and informed citizens:

$$
\begin{aligned}
\frac{d \tau_{u}}{d p}= & f\left(p r\left(m_{A}^{*}\right)\right)\left[r\left(m_{A}^{*}\right)+p r^{\prime}\left(m_{A}^{*}\right) \frac{d m_{A}^{*}}{d p}\right]= \\
& \frac{f\left(p r\left(m_{A}^{*}\right)\right)}{\Delta^{*}}\left[r\left(m_{A}^{*}\right) \Delta^{*}-p r^{\prime}\left(m_{A}^{*}\right) \Gamma^{*}\right] .
\end{aligned}
$$

Assumptions 2 and 3 imply that the above expression is negative. ${ }^{13}$

$$
\frac{d \tau_{u}}{d q}=f\left(p r\left(m_{A}^{*}\right)\right) p r^{\prime}\left(m_{A}^{*}\right) \frac{d m_{A}^{*}}{d q}<0
$$

\footnotetext{
${ }^{13}$ The expression in the brackets of (13) can be written as $\left[p q r^{\prime}\left(m_{A}^{*}\right) r^{\prime}\left(m_{B}^{*}\right) f\left(r\left(m_{B}^{*}\right)\right) r\left(m_{B}^{*}\right)-\right.$
} $\left.(1-p) q r^{\prime}\left(m_{A}^{*}\right) r^{\prime}\left(m_{B}^{*}\right) f\left(r\left(m_{A}^{*}\right)\right) r\left(m_{A}^{*}\right)\right]+\left[(1-p) r\left(m_{A}^{*}\right) r^{\prime}\left(m_{B}^{*}\right)-p r\left(m_{B}^{*}\right) r^{\prime}\left(m_{A}^{*}\right)\right]$. Since $p \geq 1 / 2$, the sum of the first two terms is positive by Assumption 2 and the sum of the last two is positive by Assumption 3. 


$$
\begin{gathered}
\frac{d \tau_{I}}{d p}=f\left(r\left(m_{I}^{*}\right)\right) r^{\prime}\left(m_{I}^{*}\right) \frac{d m_{I}^{*}}{d p}\left\{\begin{array}{c}
<0 \text { if } I=A \\
>0 \text { if } I=B
\end{array},\right. \\
\frac{d \tau_{I}}{d q}=f\left(r\left(m_{I}^{*}\right)\right) r^{\prime}\left(m_{I}^{*}\right) \frac{d m_{I}^{*}}{d q}<0 .
\end{gathered}
$$

Condition (8) is not enough to assure that, $m_{A}^{*}$, the solution to (7), is part of a desirable mixing equilibrium. For this, there also need to exist a mixing probability $\alpha \in[1 / 2,1]$ that satisfies (1), that is, that makes the realized margins of victory consistent with the citizens' behaviours and expectations. The mixing probability consistent with (1) is:

$$
\alpha=\frac{1}{2}+\frac{m_{A}-q F\left(r\left(m_{A}\right)\right)}{2(1-q) F\left(p r\left(m_{A}\right)\right)} .
$$

Thus, we need

$$
\begin{aligned}
m_{A}^{*}-q F\left(r\left(m_{A}^{*}\right)\right) & \geq 0, \\
m_{A}^{*}-q F\left(r\left(m_{A}^{*}\right)\right)-(1-q) F\left(p r\left(m_{A}^{*}\right)\right) & \leq 0 .
\end{aligned}
$$

It is easy to verify that only the second inequality matters, as the first is implied by (7) and the facts that $m_{A}^{*}>m_{B}^{*}$ and $m-q F(r(m))$ is increasing in $m$, for $m>0$.

To sum up the above analysis, a desirable mixing equilibrium exists if the boundary inequality (8) is satisfied, which implies that the equilibrium condition (7) has a solution, and, in addition, if such solution satisfies (19), which ensures the existence of a valid mixing probability. We now discuss this last condition according to the proportion of informed voters, $q$.

When the probability of candidate $A$ being the best candidate, $p$, is exactly $1 / 2$ then, by the mixing condition (5), for any $q, m_{B}\left(m_{A}\right)=m_{A}$ and, from the equilibrium condition (7) we have $m_{A}=q F\left(r\left(m_{A}\right)\right)$. In addition, we also know by (6) that $m_{0}=0$ and that, therefore, (8) is satisfied and the equilibrium equation has a solution $m_{A}^{*}$. Substituting this solution into (19) makes it

$$
-(1-q) F\left(\operatorname{pr}\left(m_{A}^{*}\right)\right) \leq 0
$$

which clearly holds for all $q \in[0,1]$. Thus, when $p=1 / 2$ a unique desirable mixing equilibrium exists for all $q \in[0,1]$.

We now consider the case where $p=\bar{p}(q)$, the maximum possible value for (8) to be satisfied. In this case, (8) holds as equality, and the solution to (7) is $m_{A}^{*}=m_{0}$. Thus, (19) becomes

$$
q F(r(0))-(1-q) F\left(\bar{p}(q) r\left(m_{0}\right)\right) \leq 0
$$

or, using (5)

$$
q F(r(0))-(1-q) F((1-\bar{p}(q)) r(0)) \leq 0
$$


Rewriting, gives us

$$
q \leq \frac{F((1-\bar{p}(q)) r(0))}{F((1-\bar{p}(q)) r(0))+F(r(0))} .
$$

Recalling from page 13 that $\bar{p}(q)$ is increasing in $q$, we have that the right hand side of the above condition is decreasing in $q$. In the meantime, the condition is satisfied at $q=0$, where $\bar{p}(0)=1 / 2$ and the right hand side is positive; it is not satisfied at $q=1 / 2$, as the right hand side is always less than $1 / 2$. By continuity, there exists a $\bar{q}<1 / 2$, such that the above condition is satisfied whenever $q \leq \bar{q}$. We just showed that for $q \leq \bar{q}$, the condition (19) for having $\alpha \leq 1$, is satisfied at $\bar{p}(q)$. It is easy to show that under assumptions 2 and 3 it is also satisfied for all $p \leq \bar{p}(q) \cdot{ }^{14}$

Consider now $q>\bar{q}$. When $p=1 / 2$, the left hand side of (19) is less than zero and when $p=\bar{p}(q)$ it is greater than zero. By monotonicity, there exists a $\widehat{p}(q) \in[1 / 2, \bar{p}(q))$ such that $(19)$ is satisfied for all $p \in[1 / 2, \widehat{p}(q)]$.

In the extreme case where $q=1$, when all citizens are informed, the equilibrium conditions (1) and (2) become $m_{A}=F\left(r\left(m_{A}\right)\right)$ and $m_{B}=F\left(r\left(m_{B}\right)\right)$. Thus, candidates $A$ and $B$ have the same winning margin in their corresponding state. A hypothetical uninformed voter would strictly prefer to vote for $A$ if $p>1 / 2$. Hence, when $q=1$, the mixing equilibrium is possible only when $p=1 / 2$ and therefore $\widehat{p}(1)=1 / 2 .{ }^{15}$

The above discussion on the conditions that must be satisfied in a mixed desirable equilibrium as well as of the definition of the thresholds $\bar{p}(q), \widehat{p}(q)$ and $\bar{q}$ leads to the following characterization of the desirable mixing equilibrium:

Proposition 1. A desirable mixing equilibrium exists if and only if, $p \in[1 / 2, \bar{p}(q))$ and $q \leq \bar{q}$, or $p \in[1 / 2, \widehat{p}(q)]$ and $q>\bar{q}$.

\subsubsection{Undesirable mixing equilibrium}

In an undesirable mixing equilibrium both candidates receive votes from uninformed voters but candidate $B$ is never elected. In order for this to occur, we need the following mixing condition

$$
\operatorname{pr}\left(m_{A}\right)=(1-p) r\left(m_{B}^{-}\right),
$$

where $r\left(m_{B}^{-}\right)$indicates that in this type of equilibrium the margin of victory for candidate $B$ must be negative. It follows that while $r\left(m_{A}\right)=r_{+}\left(m_{A}\right)$, as in $(5)$, now $r\left(m_{B}\right)=$ $r_{-}\left(m_{B}\right)$.

\footnotetext{
${ }^{14}$ To see this, notice that the derivative of the LHS of (19) with respect to $p, X_{A} \frac{d m_{A}^{*}}{d p}-(1-q) \frac{d \tau_{u}}{d p}$ is positive, as under Assumptions 2 and $3, \frac{d m_{A}^{*}}{d p}>0$ and $\frac{d \tau_{u}}{d p}<0$.

${ }^{15}$ Without further assumptions we cannot know if $\widehat{p}(q)$ is decreasing in all its domain. It can be showed that sufficient conditions for this is that the functions $F$ and $r$ are convex on $\mathrm{R}+$.
} 
The threshold $m_{0} \in[0,1]$ is defined as before in (6) and implies that for $m_{A} \in\left[m_{0}, \bar{m}\right]$ there is a unique $m_{B}^{-}$satisfying (21). We use $m_{B}^{-}\left(m_{A}\right)$ to denote this value.

Lemma 4. The function $m_{B}^{-}(\cdot)$ satisfies the following properties:

1. $m_{B}(\cdot)$ is decreasing in $m_{A}$ (weakly when $m_{A} \geq \bar{m}$ )

2. $m_{B}\left(m_{0}\right)=0$ and $m_{B}\left(m_{A}\right)=-1$, for $m_{A} \geq \bar{m}$.

3. $m_{B}(\cdot)$ is increasing $p$.

Proof of Lemma 4: Part 1 and 3:Applying the IFT to (21), recalling that $r^{\prime}(m)>0$, for $m<0$, we have

$$
\begin{aligned}
\frac{\partial m_{B}^{-}}{\partial m_{A}} & =-\frac{p r^{\prime}\left(m_{A}\right)}{-(1-p) r^{\prime}\left(m_{B}^{-}\right)}<0 \\
\frac{\partial m_{B}}{\partial p} & =\frac{r\left(m_{B}\right)+r\left(m_{A}\right)}{(1-p) r^{\prime}\left(m_{B}^{-}\right)}>0
\end{aligned}
$$

Part 2 follows from Part 1, the definition of $m_{0},(21)$ and the assumptions that $r\left(m_{A}\right)=$ 0 for $m_{A} \geq \bar{m}$.

Substituting $m_{B}^{-}\left(m_{A}\right)$ into (3) we have

$$
m_{A}-q F\left(r\left(m_{A}\right)\right)=-\left[m_{B}^{-}\left(m_{A}\right)-q F\left(r\left(m_{B}^{-}\left(m_{A}\right)\right)\right)\right] .
$$

We are interested in existence and uniqueness of a solution $m_{A}^{* *} \in\left[m_{0}, \bar{m}\right]$ to the above equation. When $m_{A}=m_{0}$, by Lemma 4 we have $m_{B}^{-}=0$. The LHS is $m_{0}-q F\left(r\left(m_{0}\right)\right)$ and the RHS is $q F(r(0))$. When $m_{A}=\bar{m}$, by Lemma 4 we have $m_{B}^{-}=-1$. The LHS is $\bar{m} \leq 1$ and the RHS is 1 . We also know that the LHS is increasing with respect to $m_{A}$ but we do not know whether the RHS is or not. However, by the intermediate value theorem we know that a sufficient condition for an intersection $m_{A}^{* *} \in\left[m_{0}, \bar{m}\right]$ to exist is

$$
m_{0}-q F\left(r\left(m_{0}\right)\right) \geq q F(r(0)) .
$$

The terms in this condition are the same as the ones defining $\bar{p}(q)$ for the desirable mixing equilibrium, (8). Now however we need $p \geq \bar{p}(q)$. It should be noticed that, while in the desirable mixing equilibrium the solution to (7) is unique, for the undesirable mixing equilibrium without further assumptions, we cannot say the same about the solution to (22). 
We continue the analysis of the undesirable mixing equilibrium under the assumption that $m_{A}^{* *}$ is unique, as it is the case for example if $f$ is uniform and the regret function is linear. ${ }^{16}$

It should be noted at this point that the expressions for the effect of $p$ and $q$ on the margins of victory and turnout in an undesirable mixing equilibrium are the same as those for the desirable case, expressions (9) through (16), provided that we replace $m_{A}^{*}$ and $m_{B}\left(m_{A}\right)$ with $m_{A}^{* *}$ and $m_{B}^{-}\left(m_{A}\right)$, respectively. For simplicity, we slightly abuse notation and use the same symbol $\Delta$ in the undesirable mixing case, but it is defined as

$$
\Delta=(1-p) r^{\prime}\left(m_{B}^{-}\left(m_{A}\right)\right) X_{A}+p r^{\prime}\left(m_{A}\right) X_{B}
$$

In order for $m_{A}^{* *}$ to be part of an undesirable mixing equilibrium, in addition to satisfying the mixing condition (21) and the coherency of anticipated and realized margins induced by behaviours (22), it must induce a mixing probability $\alpha \in[1 / 2,1]$ satisfying (1):

$$
\begin{aligned}
m_{A}^{* *}-q F\left(r\left(m_{A}^{* *}\right)\right) & \geq 0, \\
m_{A}^{* *}-q F\left(r\left(m_{A}^{* *}\right)\right)-(1-q) F\left(p r\left(m_{A}^{* *}\right)\right) & \leq 0 .
\end{aligned}
$$

Once again, it is only the last condition that matters. This is because $m_{A}^{* *}-q F\left(r\left(m_{A}^{* *}\right)\right)$ is increasing in $p$, and for $p \geq \bar{p}(q)$ this expression is positive, as at $\bar{p}(q)$ it becomes $m_{0}-q F\left(r\left(m_{0}\right)\right)$, which by definition equals $q F(r(0))>0$.

For what concerns the second condition: when $p=\bar{p}(q)$ we have $m_{0}-q F\left(r\left(m_{0}\right)\right)=$ $q F(r(0))$ and $m_{A}^{* *}=m_{0}$. Condition (25) becomes $q F(r(0))-(1-q) F((1-\bar{p}(q)) r(0)) \leq 0$ which is the same as (20). This means that condition $(25)$ is satisfied at $\bar{p}(q)$ for $q \leq \bar{q}$. However, it is not necessarily satisfied for all $p>\bar{p}(q)$. If $m_{A}^{* *}-q F\left(r\left(m_{A}^{* *}\right)\right)-(1-$ q) $F\left(\operatorname{pr}\left(m_{A}^{* *}\right)\right)$ is increasing in $p$, there exists a $\widehat{\widehat{p}}(q) \in[\bar{p}(q), 1]$ such that $(25)$ holds for $p \in[\bar{p}(q), \widehat{\widehat{p}}(q)]$. We know that $\widehat{\widehat{p}}(\bar{q})=\bar{p}(\bar{q})$, but a priori we do not know its pattern. For $q>\bar{q},(20)$ is never satisfied at $\bar{p}(q)$, and, if $m_{A}^{* *}-q F\left(r\left(m_{A}^{* *}\right)\right)-(1-q) F\left(\operatorname{pr}\left(m_{A}^{* *}\right)\right)$ is increasing in $p$, it is not satisfied for any $p>\bar{p}(q)$. It follows that, in this situation there cannot be an undesirable mixing equilibrium for $q>\bar{q}$. A sufficient condition for $m_{A}^{* *}-q F\left(r\left(m_{A}^{* *}\right)\right)-(1-q) F\left(p r\left(m_{A}^{* *}\right)\right)$ to be increasing in $p$ is that turnout of uninformed citizens $F\left(\operatorname{pr}\left(m_{A}^{* *}\right)\right)$ is decreasing in $p$. To assure that this is the case we impose the following assumptions:

\footnotetext{
${ }^{16}$ In the paper we refer to the regret function as linear in the sense of piece-wise linear of the following form: $r_{-}(m)=1+m ; r_{+}(m)=1-\gamma \cdot m$, for $m \in[0, \bar{m}]$, and $r_{+}(m)=0$, for $m>\bar{m}$, with $\gamma>1$ and $\bar{m}=1 / \gamma$.
} 
Assumption 4. (i) $X_{B}>0$; and (ii) $\Delta<0$, for all $p \geq \bar{p}(q)$.

Assumption 4 imposes restrictions only when the margin $m_{B}$ is negative. Part (i) will be used also in the analysis of undesirable pure-strategy equilibria and it is necessary for Part (ii) to hold. We have seen in the previous section that for any positive margin $m_{B}$ satisfying the mixing condition (5), $\Delta$ is always negative. Part (ii) of assumption 4 imposes that $\Delta$ be negative for all negative margins $m_{B}$ satisfying the mixing condition, only in the regions of parameters $(p$ and $q$ ) where an undesirable mixing equilibrium can possibly exist. This assumption is satisfied, for example, when $f$ is uniform and the regret function linear. In this situation the expression $\frac{d \tau_{u}}{d p}$ in (13) is negative. ${ }^{17}$ Recalling the explanation for $\Delta$ from page 14, Part (ii) implies that, in an undesirable equilibrium, an increase in the anticipated margin $m_{A}$ causes the difference between the sum of anticipated and realized winning margins in the two states to go down.

The above considerations lead us to the following characterization of the undesirable mixing equilibrium under the assumptions that the solution $m_{A}^{* *}$ to $(22)$ is unique and assumption 4 .

Proposition 2. There exists an undesirable mixed-strategy equilibrium if and only if $q<\bar{q}$ and $p \in(\bar{p}(q), \widehat{\widehat{p}}(q)]$.

\subsection{Pure-strategy equilibria}

In any pure-strategy equilibrium of this model uninformed voters vote for candidate $A$ with probability 1 , that is, $\alpha=1$. Then, (1) and (2) become:

$$
\begin{aligned}
& m_{A}-q F\left(r\left(m_{A}\right)\right)=(1-q) F\left(\operatorname{pr}\left(m_{A}\right)\right), \\
& m_{B}-q F\left(r\left(m_{B}\right)\right)=-(1-q) F\left(\operatorname{pr}\left(m_{A}\right)\right) .
\end{aligned}
$$

For $m_{A}>0$, the left-hand side of (26) is continuous and increasing in $m_{A}$ (strictly so for $m_{A}<\bar{m}$ ) and achieves its minimum (negative value) at 0 and maximum (one) at 1 . The right-hand side of (26) is positive, continuous and strictly decreasing in $m_{A}$, and achieves a minimum of zero . Therefore, (26) has a unique solution, $m_{A}^{p}>0$, where the superscript $p$ stands for pure strategy.

\footnotetext{
${ }^{17}$ Using (13), evaluated at $m_{A}^{* *}, m_{B}^{* *}$ instead of $m_{A}^{*}, m_{B}^{*}$ we have that, the expression for $\frac{d \tau_{u}}{d p}$ in the undesirbale mixing equilibrium, when $\Delta<0$, is negative if the term in brackets in (13) is positive. Such term, already developed in footnote 13 , can be re-written as $-p r^{\prime}\left(m_{A}^{* *}\right) r\left(m_{B}^{* *}\right) X_{B}^{* *}+(1-p) r\left(m_{A}^{* *}\right) r^{\prime}\left(m_{B}^{* *}\right) X_{A}^{* *}$, where $X_{i}^{* *}$ corresponds to $X_{i}^{* *}$ evaluated at $m_{i}^{* *}$, which under assumption 4. i is positive.
} 
From (26), we can show that

$$
\begin{aligned}
& \frac{d m_{A}^{p}}{d q}=-\frac{F\left(p r\left(m_{A}^{p}\right)\right)-F\left(r\left(m_{A}^{p}\right)\right)}{Y_{A}}>0, \\
& \frac{d m_{A}^{p}}{d p}=-\frac{-(1-q) f\left(p r\left(m_{A}^{p}\right)\right) r\left(m_{A}^{p}\right)}{Y_{A}}>0,
\end{aligned}
$$

where

$$
Y_{A}=X_{A}-(1-q) p f\left(\operatorname{pr}\left(m_{A}^{p}\right)\right) r^{\prime}\left(m_{A}^{p}\right)>1 .
$$

The above results in turn imply that $(1-q) F\left(\operatorname{pr}\left(m_{A}^{p}\right)\right)$ is decreasing in $q$ and increasing in $p$. The former is because both $(1-q)$ and $F\left(\operatorname{pr}\left(m_{A}^{p}\right)\right)$ decrease as $q$ increases. The latter uses (26) and the fact that its left-hand side is increasing in $m_{A}$. Therefore, we have that in a pure strategy equilibrium turnout of uninformed citizens increases with $p$ :

$$
\frac{d \tau_{u}}{d p}=\frac{d F\left(p r\left(m_{A}^{p}\right)\right)}{d p}=\frac{d m_{A}^{p}}{d p} \frac{X_{A}}{(1-q)}>0 .
$$

For what concerns the response of turnout of informed citizens to $p$ and of turnout of each group of citizens with respect to $q$, (14) through (16) are also valid in the purestrategy equilibrium case, provided that $m_{I}^{*}$ is replaced in each term with $m_{I}^{P}, I=A, B$.

Notice that a solution $m_{B}^{p}\left(m_{A}^{p}\right) \in[-1,1]$ to $(27)$ always exists, as the LHS is continuous with value on the compact set $[-1,1]$ and the RHS has value in $[-(1-q), 0]$. Assumption 4 implies that $m-q F(r(m))$ is increasing in $m$, also for $m<0$, which in turn assures that the solution $m_{B}^{p}\left(m_{A}^{p}\right)$ is unique. The following graph (drawn assuming uniform cost distribution and linear regret function) can be used to understand how $m_{A}^{p}$ and $m_{B}^{p}$ are determined.

Using the graph and the above results we have: ${ }^{18}$

$$
\begin{gathered}
\frac{d m_{B}^{p}}{d q}=\frac{\partial m_{B}^{p}}{\partial m_{A}^{p}} \cdot \frac{d m_{A}^{p}}{d q}+\left.\frac{\partial m_{B}}{\partial q}\right|_{m_{A}^{P}}= \\
\frac{-p r^{\prime}\left(m_{A}\right)(1-q) f\left(p r\left(m_{A}\right)\right)}{X_{B}} \frac{F\left(r\left(m_{A}^{p}\right)\right)-F\left(p r\left(m_{A}^{p}\right)\right)}{Y_{A}}+\frac{F\left(p r\left(m_{A}^{p}\right)\right)+F\left(r\left(m_{B}^{p}\right)\right)}{X_{B}}>0
\end{gathered}
$$

and

$$
\frac{d m_{B}^{p}}{d p}=\frac{\partial m_{B}^{p}}{\partial m_{A}^{p}} \cdot \frac{d m_{A}^{p}}{d p}+\left.\frac{\partial m_{B}}{\partial p}\right|_{m_{A}^{P}}=
$$

\footnotetext{
${ }^{18}$ The expression $\frac{\partial m_{B}^{p}}{\partial m_{A}^{p}}$ is the partial derivative of $m_{B}^{p}$ with respect to $m_{A}^{p}$ calculated from (27) holding $p$ and $q$ fixed.
} 


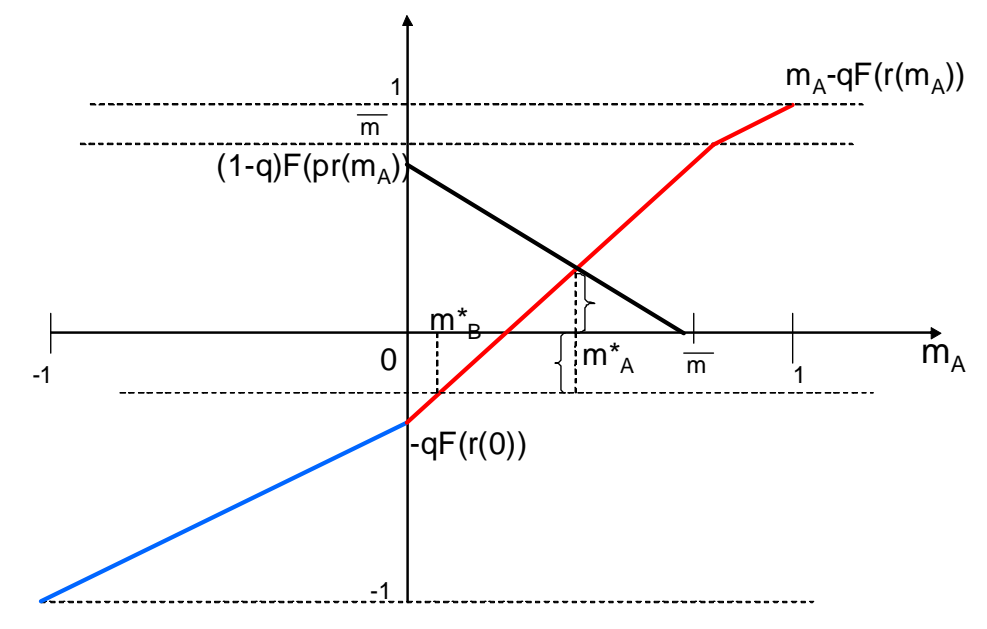

Figure 1: Pure Strategy margins

$$
\begin{aligned}
& \frac{-p r^{\prime}\left(m_{A}\right)(1-q) f\left(p r\left(m_{A}\right)\right)}{X_{B}} \frac{(1-q) f\left(p r\left(m_{A}\right)\right) r\left(m_{A}\right)}{Y_{A}}-\frac{(1-q) f\left(p r\left(m_{A}\right)\right) r\left(m_{A}\right)}{X_{B}} \\
= & \frac{-(1-q) f\left(p r\left(m_{A}\right)\right) r\left(m_{A}\right)}{X_{B} Y_{A}} X_{A}<0
\end{aligned}
$$

The solution $m_{B}^{p}\left(m_{A}^{p}\right)$ to $(27)$ is (weakly) positive, i.e., the potential pure-strategy equilibrium is desirable, if and only if

$$
(1-q) F\left(\operatorname{pr}\left(m_{A}^{p}\right)\right) \leq q F(r(0))
$$

Clearly when $q \geq 1 / 2$ condition (33) always holds. This is because, taking into consideration that informed citizens have higher turnout, uninformed citizens are not enough to counterbalance the voting choices of informed voters. For smaller proportions of informed voters, we have to analyze condition (33) further. We know that $(1-q) F\left(\operatorname{pr}\left(m_{A}^{p}\right)\right)$ is decreasing in $q$, while clearly $q F(r(0))$ is increasing. When $q=0$, LHS $>$ RHS and the opposite holds when $q=1 / 2$. It follows that there exists $\widetilde{q}(p) \in(0,1 / 2)$ such that, for all $q \geq \widetilde{q}(p),(33)$ holds, with equality at $\widetilde{q}(p)$. Notice that $\widetilde{q}(p)$ is increasing in $p$, because $(1-q) F\left(\operatorname{pr}\left(m_{A}^{p}\right)\right)$ is decreasing in $q$ and increasing in $p$. Therefore, there exists a $\widetilde{p}(q)$ such that for $p<\widetilde{p}(q)$, (33) holds. In particular at $q=\bar{q}$ we have that $\widetilde{p}(\bar{q})=\widehat{p}(\bar{q})$. This is because at $\bar{q}$ and $\widehat{p}(\bar{q})$ there is a desirable mixing equilibrium with $\alpha=1$, where $m_{A}^{p}=m_{0}$ and $m_{B}^{p}=0$. Notice also that there exists a unique $\widetilde{q} \in(\bar{q}, 1 / 2)$ such that $\widetilde{p}(q)=1$ for all $q \geq \widetilde{q}$. In addition, $\widetilde{q}<\min \{1, \bar{m} / F(r(0))\}$. This is because when $p=1$, the equation 
defining $m_{A}^{p}$ becomes $m_{A}^{p}=F\left(r\left(m_{A}^{p}\right)\right)$ and $\widetilde{q}$ is the solution to $(1-q) m_{A}^{p}=q F(r(0))$, that is $\widetilde{q}=m_{A}^{p} /\left(m_{A}^{p}+F(r(0))\right)$, which is always smaller than $\min \{1, \bar{m} / F(r(0))\}$.

Condition (33) is necessary but not sufficient for having a desirable pure strategy equilibrium. In fact a pure-strategy equilibrium does not exist if, at the given margins, uninformed citizens strictly prefer to vote for candidate $B$. The following Lemma, are important for the characterization of pure-strategy equilibrium.

Lemma 5. For $q<\bar{q}, \widetilde{p}(q)<\bar{p}(q)$; for $q=\bar{q}, \widetilde{p}(q)=\bar{p}(q)$; and for $q>\bar{q}, \widetilde{p}(q)>\bar{p}(q)$.

\section{Proof of Lemma 5:}

For $q=\bar{q}$ follows directly from the fact that at $\bar{q}$ we have $\bar{p}(\bar{q})=\widehat{p}(\bar{q})$. At $\widehat{p}, m_{A}^{*}$ satisfying (7) is such that equation (1) is satisfied with $\alpha=1$. At $\bar{p}, m_{B}^{*}=0$. Since there is a unique $m_{A}(p, q, \alpha)>0$ that solves $(1)$, at $\bar{p}(q)=\widehat{p}(\bar{q}), m_{A}^{*}=m_{A}^{p}$ and $m_{B}^{*}=m_{B}^{p}=0$.

For $q<\bar{q}$ : fix a $q$ and the corresponding $\bar{p}(q)$. We have $m_{A}^{*}=m_{0}, m_{B}^{*}=0$ and the $\alpha$ satisfying (1) is strictly less than 1 . For the same $(q, \bar{p}(q))$ consider the $m_{A}^{p}$ and $m_{B}^{p}$ satisfying (1) and (2), respectively, with $\alpha=1$. Since, under assumptions 2 and 3, $m_{A}(p, q, \alpha)$ is increasing in $\alpha$, we have that $m_{A}^{p}>m_{A}^{*}$. In addition, since $m_{A}^{p}$ and $m_{B}^{p}$ must also satisfy (3), and $m-q F(r(m))$ is increasing in $m$ for $m>0$, it must be that $m_{B}^{*}>m_{B}^{p}$. It follows that at $\bar{p}(q), m_{B}^{p}<0$, and therefore, since $m_{B}^{p}$ is decreasing in $p, \widetilde{p}(q)$ must be smaller than $\bar{p}(q)$.

For $q>\bar{q}$ the proof is analogous. For this range of $q$, we have that $\bar{p}(q)>\widehat{p}(q)$, which implies that along $\bar{p}(q)$, the $\alpha$ that satisfies (1) at the $m_{A}^{*}$ satisfying (7) is some $\alpha>1$. For the same reason as above this now implies that $m_{A}^{p}$ and $m_{B}^{p}$ satisfying (1) and (2) are such that $m_{A}^{p}<m_{A}^{*}$ and $m_{B}^{p}>m_{B}^{*}=0$. Therefore, since $m_{B}^{p}$ is decreasing in $p, \widetilde{p}(q)$ must be greater than $\bar{p}(q)$.

The results in Lemma 5 imply that for low $q$ the threshold for mixing $\widehat{\widehat{p}}(q)$ is above the thresholds for desirable pure-strategies $\widetilde{p}(q)$, while the opposite is true for higher $q .{ }^{19}$ The representation of the different thresholds is illustrated in Figure 2.

Lemma 6. For any fixed $q$ : (i) $d\left(p r\left(m_{A}^{p}\right)\right) / d p>0$ and (ii) $d\left((1-p) r\left(m_{B}^{p}\right)\right) / d p<0$, where $\left(m_{A}^{p}, m_{B}^{p}\right)$ are the solutions to (26) and (27).

\section{Proof of Lemma 6:}

\footnotetext{
${ }^{19}$ For $\mathrm{q}<\bar{q}$ we have shown on page 18 that $\widehat{\hat{p}}>\bar{p}$. Similarly, for $q>\bar{q}$ we have shown on page 16 that $\widehat{p}<\bar{p}$.
} 
Part (i) follows from (30) and the fact that $\tau_{u}$ is increasing in $\operatorname{pr}\left(m_{A}^{p}\right)$. For part (ii) we have that $d\left((1-p) r\left(m_{B}^{p}\right)\right) / d p=-r\left(m_{B}^{p}\right)+(1-p) r^{\prime}\left(m_{B}^{p}\right) \frac{d\left(m_{B}^{p}\right)}{d p}=\frac{1}{X_{B} Y_{A}}\left(-r\left(m_{B}^{p}\right) X_{A} X_{B}+(1-\right.$ q) $\left.f\left(p r\left(m_{A}^{p}\right)\right)\left[\operatorname{pr}\left(m_{B}^{p}\right) r^{\prime}\left(m_{A}^{p}\right) X_{B}-(1-p) r^{\prime}\left(m_{B}^{p}\right) r\left(m_{A}^{p}\right) X_{A}\right]\right)$. The first term in parenthesis is negative, so a sufficient condition for whole expression to be negative is that the term in squared bracket is negative. When $m_{B}^{p}<0, r^{\prime}\left(m_{B}^{p}\right)>0$, so, under assumption 4, $\operatorname{pr}\left(m_{B}^{p}\right) r^{\prime}\left(m_{A}^{p}\right) X_{B}<0$, and $-(1-p) r^{\prime}\left(m_{B}^{p}\right) r\left(m_{A}^{p}\right) X_{A}<0$. To see that the term in squared bracket is negative also when $m_{B}^{p} \geq 0$, rewrite it as the sum of the following two terms, $\left[(1-p) r\left(m_{A}^{p}\right) r^{\prime}\left(m_{B}^{p}\right)-p r\left(m_{B}^{p}\right) r^{\prime}\left(m_{A}^{p}\right)\right]+\left[p q r^{\prime}\left(m_{A}^{p}\right) r^{\prime}\left(m_{B}^{p}\right) f\left(r\left(m_{B}^{p}\right)\right) r\left(m_{B}^{p}\right)-\right.$

$\left.(1-p) q r^{\prime}\left(m_{A}^{p}\right) r^{\prime}\left(m_{B}^{p}\right) f\left(r\left(m_{A}^{p}\right)\right) r\left(m_{A}^{p}\right)\right]$, that we already met in footnotes 13 and 17 to prove the sign of $\frac{d \tau_{u}}{d p}$ in the mixing equilibria. The first term is negative, by assumption 3 and the fact that $p \geq 1 / 2$, and the second term is negative by assumption $2 . \square$

An implication of Lemma 6 is that mixed-strategy and pure-strategy equilibria are mutually exclusive, that is, for any $q$, the thresholds $\widehat{\hat{p}}(q)$ and $\widehat{p}(q)$ below which there exists a mixed-strategy undesirable and desirable equilibrium, respectively, are the same as the thresholds above which there exists a pure-strategy equilibrium. To see this, notice first that for any $(q, p)$ there is a unique $\left(m_{A}^{p}, m_{B}^{p}\right)$ solving (26) and (27), that is solving (1) and (2) with $\alpha=1$. From the Lemma then it follows that if at some $(q, p)$ there is a pure-strategy equilibrium $\left(m_{A}^{p}, m_{B}^{p}\right)$, where by definition $\operatorname{pr}\left(m_{A}^{p}\right) \geq(1-p) r\left(m_{B}^{p}\right)$, then also at a higher $p$ there is a pure-strategy equilibrium. In addition, mixed strategy equilibria exist for relatively low $p$ and in the parameter region where there are mixing equilibria, the mixing probability $\alpha$ is increasing in $p^{20}$. This implies that as $p$ increases, $\alpha$ increases til it reaches 1 at the thresholds $\widehat{\widehat{p}}(q)$ and $\widehat{p}(q)$, for low and high $q$ respectively. Along this thresholds there is a pure-strategy equilibrium $\left(m_{A}^{p}, m_{B}^{p}\right)$ where $\operatorname{pr}\left(m_{A}^{p}\right)=(1-p) r\left(m_{B}^{p}\right)$. Lemma 6 then implies that for lower $p, \operatorname{pr}\left(m_{A}^{p}\right)<(1-p) r\left(m_{B}^{p}\right)$, and voting for $A$ with probability 1 is not optimal.

Proposition 3. For any $(p, q)$ :

(i) There exists a desirable pure-strategy equilibrium iff $q \geq \bar{q}$ and $p \in[\widehat{p}(q), \widetilde{p}(q)]$.

(ii) There exists a undesirable pure-strategy equilibrium iff $q \geq \bar{q}$ and $p \geq \widetilde{p}(q)$ or $q<\bar{q}$ and $p \geq \widehat{\widehat{p}}(q)$.

Proof of Proposition 3: (i) and (ii) follow from the definition of $\widetilde{p}(q)$, Lemma 5, Lemma 6 and its implications of mutual exclusivity of mixed- and pure-strategy equilibria.

In the situation with uniform cost distribution and linear regret, all assumptions relevant for the main propositions are satisfied: not only assumptions 1, 2, 3, 4 are satisfied,

\footnotetext{
${ }^{20}$ This can be easily seen from equation equation (17) and the fact that in mixing equilibria $m_{A}$ is increasing in $p$ and $\operatorname{pr}\left(m_{A}\right)$ is decreasing.
} 


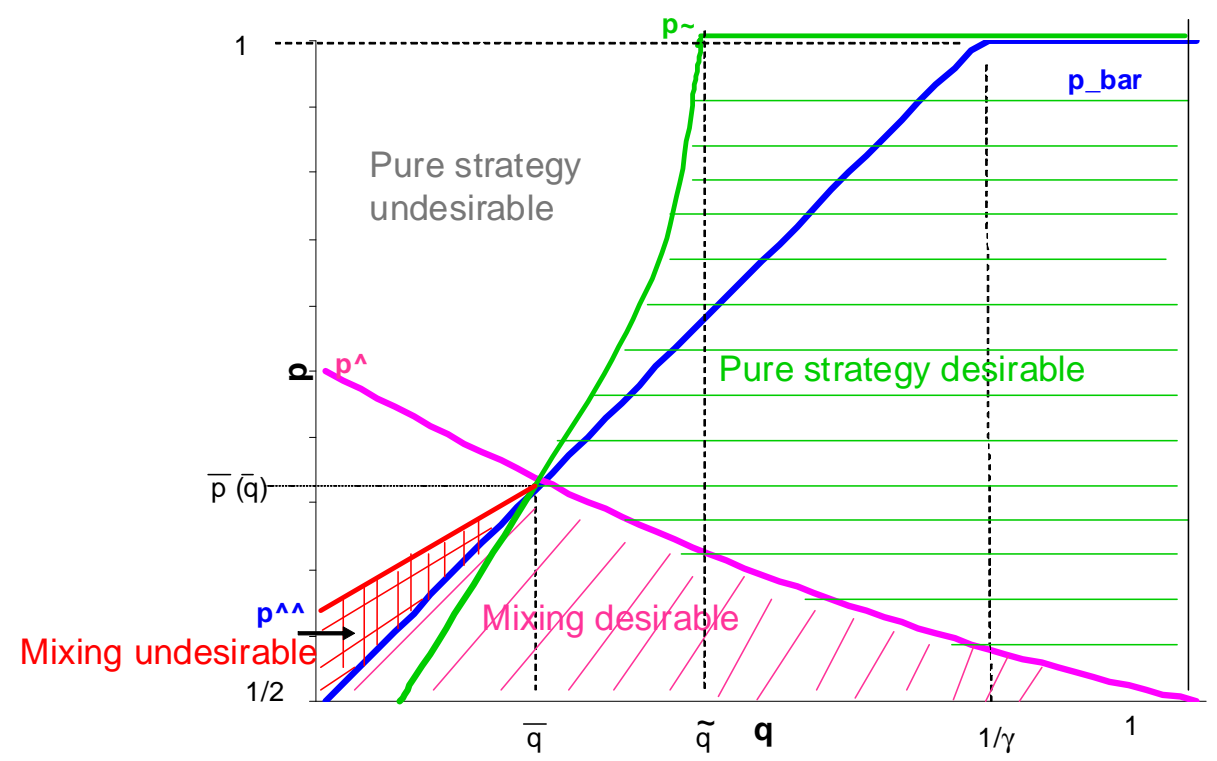

Figure 2: Equilibrium characterization

but it is easy to prove that $m_{A}^{* *}$ is unique. Figure 2 illustrates the equilibrium characterization for this case.

It is easy to verify that in all types of equilibrium $r\left(m_{B}\right)>r\left(m_{A}\right)$, that is, the equilibrium regret from not voting is higher in the ex-ante less likely state. This is obvious in mixing equilibria, where from the mixing condition $r\left(m_{B}^{*}\right)=\frac{p}{1-p} r\left(m_{A}^{*}\right)>r\left(m_{A}^{*}\right)$ for $p>1 / 2$. It is also obvious for desirable pure-strategy equilibria, where $m_{A}^{P}>m_{B}^{P} \geq 0$ and, therefore, by assumption $1, r\left(m_{B}^{P}\right)>r\left(m_{A}^{P}\right)$. In undesirable pure-strategy equilibria, where by definition $m_{A}^{P}>0>m_{B}^{P}$, it follows from the fact that equation (3) can be satisfied only for $m_{A}^{P}>-m_{B}^{P}$, which in turns implies, by assumption 1 , that $r\left(m_{B}^{P}\right)>$ $r\left(m_{A}^{P}\right)$.

We can then state that in any equilibrium, turnout of informed voters in the less likely state is higher than that in the more likely state, which in turn is higher than turnout of uninformed voters. That is, $\tau_{B}>\tau_{A}>\tau_{u}$. The finding that informed voters go to vote more than uninformed ones is consistent with many other models as well as empirical evidence. ${ }^{21}$ Our analysis also tells us that turnout should be higher in the less likely state.

\footnotetext{
${ }^{21}$ See, e.g., Feddersen and Pesendorfer [10] and Degan and Merlo [9].
} 


\section{Comparative statics}

In this section we conduct comparative statics analysis of the equilibrium with respect to $p$, the ex-ante probability that $A$ is the best candidate, and $q$, the proportion of informed citizens. Throughout the section it will be assumed that assumptions 1 through 4 hold. We start by considering the implications for the margins of victory:

Proposition 4. In the equilibrium of our psychologically-based model of voting:

1. The winning margin for each candidate is increasing in the probability of the candidate being the better one, i.e. $m_{A}$ is increasing in $p$ and $m_{B}$ is decreasing in $p$.

2. The winning margin for candidate $A$ in state $s_{A}, m_{A}$, is increasing in the proportion of informed citizens $q$ in pure-strategy and in desirable mixing equilibria, but is decreasing in undesirable mixing equilibria. The winning margin for candidate $B$ in state $s_{B}, m_{B}$, is increasing in the proportion of informed citizens $q$ in all types of equilibria.

\section{Proof or Proposition 4:}

Recalling from page 14 that expressions in (9) through (12) are valid also for the undesirable mixing equilibrium, but possibly with a different sign, and taking into consideration the results from Lemmas 3 and 4 :

1. The results follow for state $s_{A}$ from (9) and (29), and for state $s_{B}$ from (11) and (32).

2. The results follow for state $s_{A}$ from (10) and (28), and for state $s_{B}$ from (12) and $(31)$.

Proposition 4 indicates that, as the information about who the best candidate is becomes more precise, the margins of victory for the best candidate increase in all types of equilibrium. When the proportion of informed citizens increases, the effect on the margin of victory for the right candidate will depend on the realization of the state and the type of equilibrium. In all types of equilibria but the undesirable mixing, higher $q$ leads to higher margins in both states. In an undesirable mixing equilibrium the margins will go down in the ex-ante most likely state, $s_{A}$, and up in the other state, $s_{B}$.

We illustrate the intuition for the somewhat counterintuitive result that $m_{A}$ is decreasing in $q$ in the undesirable mixing equilibrium, which in turn implies that the margin $m_{A}$ is non-monotonic in $q$ for relatively low levels of $p$. Observe first that in the equilibrium condition (7), an increase in $q$ would mean that the sum of the realized margins would be too high because there are more informed voters casting votes, compared to the sum 
of the anticipated margins. The question is then in which way $m_{A}$ must be adjusted so as to restore the balance. Suppose we increase $m_{A}$. This has two effects, one direct and the other indirect. The direct effect is that since $X_{A}>0$, it would increase the difference between the anticipated margin of Candidate $A$ and the realized margin in state $A$ due to votes cast by informed voters. However, the indirect effect is that due to the indifference condition of the uninformed voters, $m_{B}$ must now become more negative since we are in an undesirable equilibrium. By assumption 4.ii, $\Delta<0$, which implies that a change in $m_{A}$ has a large effect on $m_{B}{ }^{22}$ which means that the difference between the anticipated margin of Candidate $B$ and the realized margin in state $B$ due to votes cast by informed voters is significantly decreased, so much so that it overwhelms the direct effect. So, an increase in $m_{A}$ would actually mean the realized margins would be even higher compared with the anticipated margins. Therefore, to restore equilibrium, $m_{A}$ must be decreased when the proportion of informed voters, $q$, increases.

We continue the comparative statics analysis by considering in order the effect of our two information parameters, $q$ and $p$, on turnout.

Proposition 5. In the equilibrium of our psychologically-based model of voting:

1. Turnout of uninformed citizens is decreasing in $q$ in pure-strategy and desirable mixing equilibria, but increasing in $q$ in undesirable mixing equilibria.

2. Turnout of informed citizens is decreasing in $q$ in both states in all desirable equilibria, increasing in $q$ in both states in undesirable mixing equilibria, but decreasing in $q$ in state $s_{A}$ and increasing in $q$ in state $s_{B}$ in undesirable pure-strategy equilibria.

\section{Proof or Proposition 5:}

1. The derivative of turnout of uninformed citizens with respect to $q$ is $\frac{d \tau_{u}}{d q}=f\left(\operatorname{pr}\left(m_{A}^{*}\right)\right)$ $p r^{\prime}\left(m_{A}^{*}\right) \frac{d m_{A}}{d q}$. The results follows from the sign of $\frac{d m_{A}}{d q}$ in the different types of equilibria (see part 2 of Proposition 4).

2. The derivative of turnout of informed citizens in state $I$ with respect to $q$ is $\frac{d \tau_{I}}{d q}=$ $f\left(p r\left(m_{I}\right)\right) p r^{\prime}\left(m_{I}\right) \frac{d m_{I}}{d q}$. The results follows from the sign of $\frac{d m_{I}}{d q}$ in the different types of equilibria (see part 2 of Proposition 4 )

Since turnout of informed citizens is determined by the winning margin of the right candidate, its change is directly implied by the corresponding part of Proposition 4 and assumptions on the regret function. For example, in desirable pure-strategy equilibria,

\footnotetext{
${ }^{22}$ Assumption 4 is a variation of the "winner regrets less" assumption of Li and Majumdar [22], introduced in footnote 9 .
} 


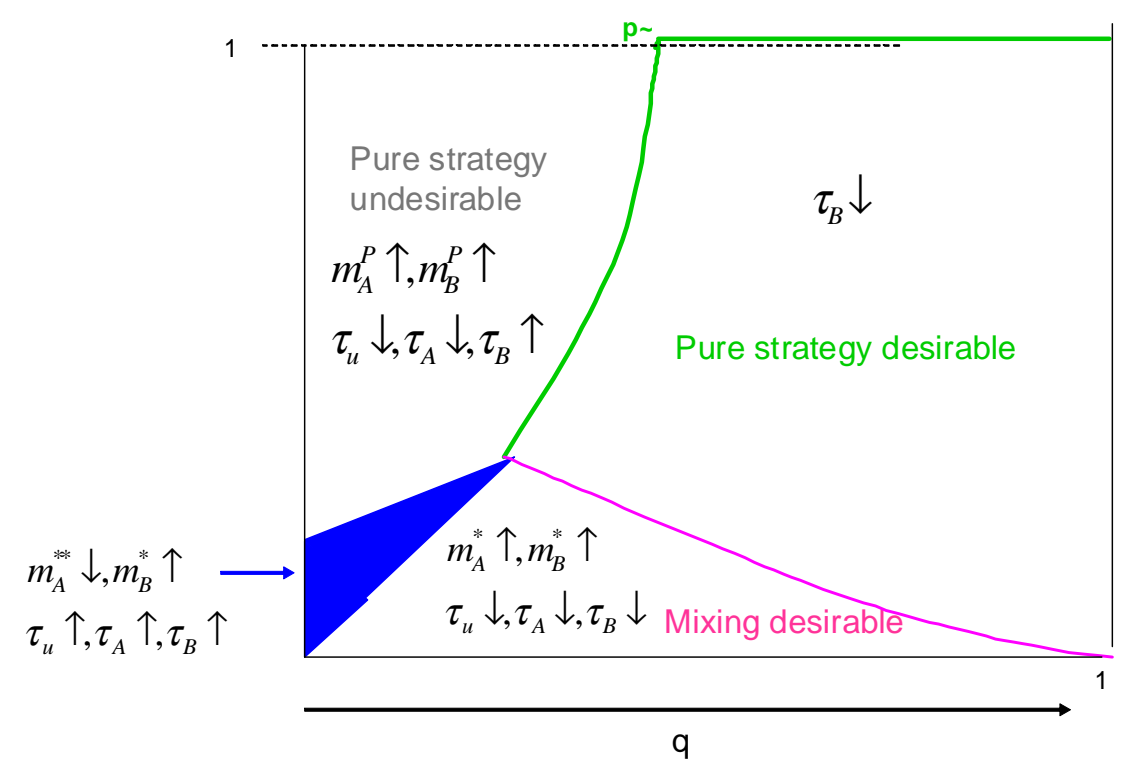

Figure 3: Comparative statics: proportion of informed q

since the winning margins of the right candidate, $A$ in $s_{A}$ and $B$ in $s_{B}$, are both increasing in $q$, the proportion of informed voters, the turnout of informed voters must be decreasing in $q$ in both states. On the other hand, in undesirable mixing equilibria, the winning margin of Candidate $A$ in $s_{A}$ is decreasing in $q$ but the winning margin of Candidate $B$ in $s_{B}$ is increasing in $q$, which implies that in both states, as $q$ increases, the winning margin moves towards 0 . Hence, the turnout of the informed voters is increasing in $q$ in both states.

The change in the turnout of uninformed citizens, on the other hand, only depends on the winning margin of Candidate $A$ in state $s_{A}$, so it is decreasing in $q$ in all types of equilibria but undesirable mixing equilibria.

Proposition 5 indicates that, the response of turnout of informed and uninformed citizens goes in the same direction in all cases except the undesirable pure-strategy equilibrium in state $\mathrm{B}$, where turnout of uninformed decreases but the one of informed increases. In addition, turnout of uninformed citizens, and that of informed (conditional on the state), can be non monotonic in $q$. This will be the case for relatively low $p$, starting with an undesirable mixing equilibrium when $q$ is low, moving to a desirable mixing equilibrium as $q$ increases, and eventually, as $q$ increases further, to a desirable pure-strategy equilibrium.

Figure 3 represents the comparative statics results with respect to the proportion of informed voters $q$. 
Proposition 6. In the equilibrium of our psychologically-based model of voting:

1. Turnout of uninformed citizens is decreasing in the ex-ante belief $p$ in mixing equilibria, while it is increasing in $p$ in pure-strategy equilibria.

2. Turnout of informed citizens in state $s_{A}$ is decreasing in $p$. Turnout of informed citizens in state $s_{B}$ is increasing in $p$ in desirable equilibria and is decreasing in $p$ in undesirable equilibria.

\section{Proof or Proposition 6:}

1. The derivative of turnout of uninformed citizens with respect to $p$ is $\frac{d \tau_{u}}{d p}=d F\left(\operatorname{pr}\left(m_{A}\right)\right) / d p$, where $m_{A}$ is the equilibrium margin. We have shown in section 3.1, that this is expression is negative in desirable and undesirable mixing equilibria (expression in (13)), and, in section 3.2, that it is positive in pure-strategy equilibria (expression in 30)).

2. The derivative of turnout of informed citizens in state $I$ with respect to $p$ is $\frac{d \tau_{I}}{d p}=$ $f\left(r\left(m_{I}\right)\right) r^{\prime}\left(m_{I}\right) \frac{d m_{I}}{d p}$. The result follows form the signs of $r^{\prime}\left(m_{I}\right)$ in the different types of equilibria and of $\frac{d m_{I}}{d p}$ in the different states (see Proposition 4). $\square$

Proposition 6 indicates that turnout of uninformed citizens is non-monotonic in $p$, the clarity of information about who the best candidate is. As it becomes increasingly likely that $A$ is the better candidate, turnout initially decreases but then it increases. We hereby offer an intuition for these results. As $p$ increases, it becomes more attractive for uninformed voters to vote for $A$. However, their response is different in a pure-strategy equilibrium than in a mixed equilibrium. In the former, they can only respond by turning out more to vote for $A$, driving up the wining margin for $A$ in state $A$ and down the winning margin for $B$ in state $B$ (consistent with Proposition 4). However, in the latter, they may switch from voting for $B$ to voting for $A$, again driving up the winning margin of $A$ in state $A$ and down the winning margin of $B$ in state $B$ (again, consistent with Proposition 4 ). Note that in a mixing equilibrium, the cost cutoff that determines turnout is equal to both the expected regret from not voting for $A, \operatorname{pr}\left(m_{A}\right)$, and that from not voting for $B,(1-p) r\left(m_{B}\right)$. In undesirable mixing equilibrium, it is immediate that the latter goes down as $p$ increases, because the winning margin $m_{B}$ becomes more negative, resulting in less expected regret for not voting for $B$. This means that turnout will necessarily go down. ${ }^{23}$ In desirable mixing equilibrium, the log-concavity assumption ensures that the net effect on $(1-p) r\left(m_{B}\right)$ is negative.

\footnotetext{
${ }^{23}$ The same intuition applies and is easier to see in the benchmark case where there are no informed voters, which means $m_{B}=-m_{A}$.
} 


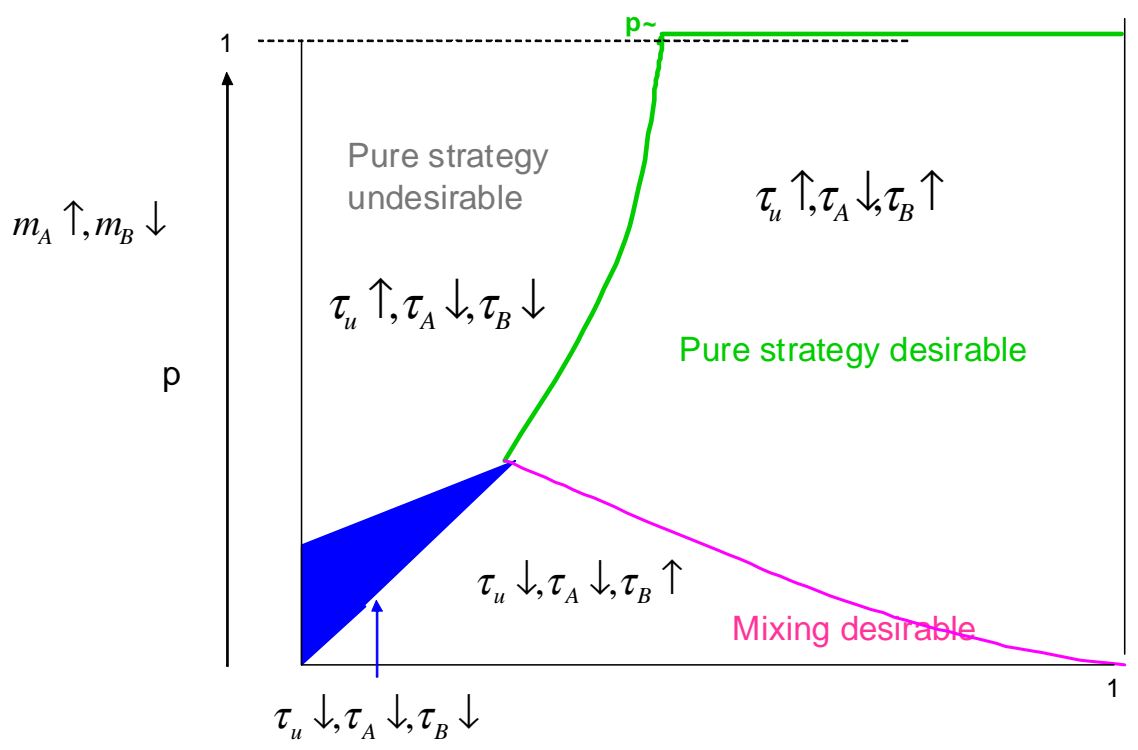

Figure 4: Comparative statics: ex-ante probability $p$

On the other hand, the response of turnout of informed citizens to $p$ is entirely driven by the anticipated margin. While in state $A$ higher $p$ always corresponds to higher margins and lower regret, in state $B$ higher $p$ corresponds to higher margins but to higher regret when the margin is negative and to lower regret when the margin is positive. This implies, that turnout of informed can go in the same (negative) or opposite direction in the two states. Interestingly, it also implies that in the less likely state, when the proportion of informed voters is relatively large, turnout of informed voters in monotonically increasing in $p$, but, when the proportion of informed citizens is relatively small, their turnout is non-monotonic in $p$. It first increases and eventually decreases.

Figure 4 represents the comparative statics results with respect to the ex-ante probability of state $A$.

The comparative statics results above can also be used to make some assessment about the relationship between the margins of victory in the different states and total turnout. Let us denote total turnout in state $s_{I}, \mathrm{I}=\mathrm{A}, \mathrm{B}$, by $T_{I}$. Its expression is:

$$
T_{I}=(1-q) \tau_{u}+q \tau_{I} .
$$

If we consider different margins of victory corresponding to different levels of information $p$, holding everything else fixed, it is easy to verify that our results indicate that there are some situations where, higher margins corresponds to lower turnout. This is 
the case, for example in desirable pure-strategy equilibria in state $s_{B}$ and in undesirable mixing-equilibria. ${ }^{24}$ Interestingly, there are situations where higher margins corresponds to higher turnout. This is the case, for example, in pure-strategy equilibria in state $s_{A} \cdot{ }^{25}$ We would obtain similar implications if different margins of victory where corresponding to different levels of $q$, holding everything else fixed.

The way winning margins affect turnout in our model resembles the way they indirectly affect turnout in pivotal-voter models, including that of Feddersen and Pesendorfer [10]. However, the implications of our model are quite different. Our comparative statics finding contrast with what happens in the pivotal-voter model of Feddersen and Pesendorfer [10], where both the margins of victory and turnout of uninformed citizens increase with the probability of being informed, and where in large elections, the prior probability concerning the state of the world plays no critical role. Our results that turnout of uninformed citizens is non-monotonic in (the ex-ante information) $p$, distinguishes our model from simple pivotal-voter models with costly voting. For example, it is easy to verify that in a finite population version of our model, with only uninformed citizens who care exclusively about the expected electoral outcome, turnout is always increasing in $p$.

\section{Conclusions}

In this paper, based on empirical evidence in elections and psychological studies that regret is an important factor of individual decision making, we have proposed a regretbased model of voting with common value, uncertainty about the state of the world, and asymmetric information. The main ingredient of the model is that citizens anticipate the regret they would experience if they fail to vote or vote for the wrong candidate and that such regret is decreasing in the margin of victory for the right candidate and increasing in the margin of victory for the wrong candidate. We have characterized the equilibrium. The proportion of informed citizens and the ex-ante likelihood of each candidate been the best one, determines whether the equilibrium is desirable, where the right candidate is always elected, or undesirable, where the wrong candidate is elected in some state. Pure-strategy equilibria, where uninformed voters always vote for the ex-ante favoured candidate, exist for relatively high probability of that candidate being the best. Desirable

\footnotetext{
${ }^{24}$ In a desirable pure-strategy equilibrium we have that $m_{B}^{P}$ is decreasing in $p$, by Proposition 4.1, while both $\tau_{u}$ and $\tau_{B}$ are increasing in $p$ by Proposition 6.1 and 6.2, respectively. Consider now an undesirable mixing equilibrium. In state $A, m_{A}^{* *}$ increases in $p$, by Proposition 4.1, while both $\tau_{u}$ and $\tau_{A}$ are decreasing in $p$ by Proposition 6.1 and 6.2 , respectively. In state $B, m_{B}^{* *}$ decreases in $p$, meaning that the margin for candidate $B$ becomes more negative, while both $\tau_{u}$ and $\tau_{B}$ are decreasing in $p$.

${ }^{25}$ From (25), we have $m_{A}=T_{A}$, so that higher equilibrium margins for candidate $A$ must correspond to higher equilibrium turnout.
} 
equilibria exist for relatively high proportion of informed citizens.

The model we propose provides a tractable framework that is on solid footing in terms of consistency with psychological studies on decision making and stylized facts about voter turnout. It delivers many interesting distinctive comparative statics results. For example, in all equilibria, turnout of informed citizens is higher in the less likely state. The winning margin for the right candidate can be increasing or decreasing in the proportion of uninformed citizens, depending on the type of equilibrium. Turnout of both informed and uninformed citizens is non monotonic in the ex-ante probability that one candidate is the correct one and in the proportion of informed citizens. The response of turnout of informed and uninformed citizens to changes in these two elements can go in different directions, depending on the state and type of equilibrium. Furthermore, winning margins can be positively or negatively correlated with total turnout.

The distinctive implications of the model can be confronted with the data using experimental or empirical studies. In addition, our comparative static analysis regarding the proportion of informed voters can be viewed as a precursor to a more involved analysis about information provision (by the government or candidates) and information acquisition (by voters).

\section{References}

[1] Marina Agranov, Jacob K. Goeree, Julian Romero, and Leeat Yariv. What makes voters turn out: The effects of polls and beliefs. mimeo, 2013.

[2] John H. Aldrich. When is it rational to vote? In Mueller D.C., editor, Social Perspectives on Public Choice. Cambridge: Cambridge University Press, 1997.

[3] David E. Bell. Regret in decision making under uncertainty. Operations Research, 30(5):961-981, 1982.

[4] Tilman Borgers. Costly voting. American Economic Review, 94(1):57-66, 2004.

[5] Pedro Chaves and Susana Peralta. A self-delusive theory of voter turnout. mimeo, 2013.

[6] Stephen Coate and Michael Conlin. A group rule-utilitarian approach to voter turnout: Theory and evidence. American Economic Review, 94(5):1476-1504, 2004.

[7] Stephen Coate, Michael Conlin, and Andrea Moro. The performance of pivotal-voter models in small-scale elections: Evidence from texas liquor referenda. Journal of Public Economics, 92(3-4):582-596, April 2008.

[8] Arianna Degan. Civic duty and political advertising. Economic Theory, 52(2):531$564,2013$.

[9] Arianna Degan and Antonio Merlo. A structural model of turnout and voting in 
multiple elections. Journal of the European Economic Association, 9(2):209-245, 2011.

[10] Timothy J. Feddersen and Wolfgang Pesendorfer. The swing voter's curse. American Economic Review, 86(3):408-424, 1996.

[11] Timothy J. Feddersen and Alvaro Sandroni. The calculus of ethical voting. International Journal of Game Theory, 35(1):1-25, 2006.

[12] Timothy J. Feddersen and Alvaro Sandroni. A theory of participation in elections. American Economic Review, 96(4):1271-1282, 2006.

[13] John Ferejohn and Morris P. Fiorina. The paradox of not voting: A decision-theoretic analysis. American Political Science Review, 68:525-536, 1974.

[14] Morris P Fiorina. The voting decision: instrumental and expressive aspects. The Journal of Politics, 38(02):390-413, 1976.

[15] Alan Hamlin and Colin Jennings. Expressive political behaviour: Foundations, scope and implications. British Journal of Political Science, 41(03):645-670, 2011.

[16] John C. Harsanyi. Rule utilitarianism, rights, obligations, and the theory of rational behavior. Theory and Decision, 12:115-133, 1980.

[17] Arye L Hillman. Expressive behavior in economics and politics. European Journal of Political Economy, 26(4):403-418, 2010.

[18] Arye L Hillman. Expressive voting and identity: evidence from a case study of a group of us voters. Public Choice, 148(1-2):249-257, 2011.

[19] Janet Landman. Regret: Persistence of the possible. New York: Oxford University Press, 1993.

[20] John O. Ledyard. The paradox of voting and candidate competition: a general equilibrium analysis. In G. Horwich and J. Quirck, editors, Essays in Contemporary Fields of Economics. Purdue University Press: West Lafayette, 1982.

[21] David K. Levine and Thomas R. Palfrey. The paradox of voter participation: a laboratory study. American Political Science Review, 101(1):143-158, 2007.

[22] Ming Li and Dipjyoti Majumdar. A psychologically based model of voter turnout. Journal of Public Economic Theory, 12(5):979-1002, 2010.

[23] Graham Loomes and Robert Sugden. Regret theory: an alternative theory of rational choice under uncertainty. The Economic Journal, 92(368):805-824, 1982.

[24] Antonio Merlo. Whither political economy? theories, facts, and issues. In Richard Blundell, Whitney K. Newey, and Torsten Persson, editors, Advances in Economics and Econometrics, Theory and Applications: Ninth World Congress of the Econometric Society. Cambridge: Cambridge University Press, 2006.

[25] Pietro Ortoleva and Erik Snowberg. Overconfidence in political behavior. mimeo, 2014. 
[26] Thomas R. Palfrey and Howard Rosenthal. Voter participation and strategic uncertainty. American Political Science Review, 79:62-78, 1985.

[27] Neal J. Roese. If Only: How to Turn Regret Into Opportunity. Broadway, 2005.

[28] Neal J Roese and James M Olson. What might have been: The social psychology of counterfactual thinking. Psychology Press, 2014.

[29] Jan Schnellenbach and Christian Schubert. Behavioral public choice: A survey. Technical report, Freiburger Diskussionspapiere zur Ordnungsökonomik, 2014.

[30] Alexander A. Schuessler. A Logic of Expressive Choice. Princeton University Press, 2000. 